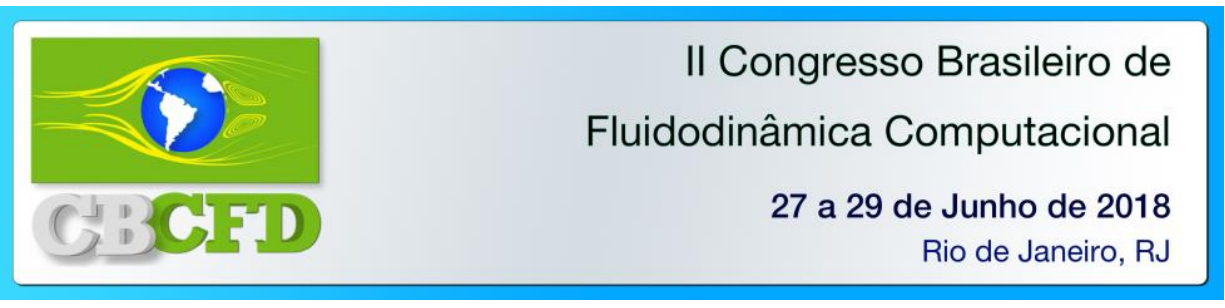

\title{
Estudo da influência do grau de submergência na hidrodinâmica de um conversor oscilante por translação de ondas
}

\author{
G. F. C. VARGAS ${ }^{1}$, E.B.C. SCHETTINI ${ }^{2}$ \\ ${ }^{1,2}$ Universidade Federal do Rio Grande do Sul - Instituto de Pesquisas Hidráulicas \\ E-mail para contato: guilherme.fuhrmeister@ufrgs.br
}

\begin{abstract}
RESUMO - Os conversores oscilantes por translação de ondas representam uma tecnologia de grande potencial de geração de eletricidade no âmbito das energias renováveis. Um dos fatores influentes relacionado à hidrodinâmica destes dispositivos consiste na avaliação do grau de submergência de suas placas, onde parte do conversor encontra-se externa à superfície da água. Para tal estudo foram utilizadas simulações numéricas baseadas na metodologia RANS de modelagem, por meio do código computacional OpenFOAM v.4.1 e a extensão OLAFOAM para a geração e hidrodinâmica das ondas regulares. O objeto de estudo consiste em um dispositivo parcialmente submerso localizado próximo à superfície livre, que tem seu grau de submergência variado. Os resultados mostraram que o melhor desempenho hidrodinâmico, em termos de amplitudes e velocidades angulares atingidas, é obtido quando o conversor encontra-se $90 \%$ submerso.
\end{abstract}

\section{INTRODUÇÃO}

Tecnologias capazes de converter a energia das ondas em energia elétrica vêm ganhando destaque no âmbito das energias renováveis, em função da capacidade dos oceanos de um fornecimento contínuo de energia. Um dos principais dispositivos conversores, que encontra-se em fase pré-comercial de desenvolvimento, baseia-se no princípio de translação das ondas, que provoca um movimento oscilatório de uma placa, a qual é responsável por mover um pistão, que bombeia um fluido em alta pressão em um sistema hidráulico secundário, movimentando uma turbina responsável pela produção de eletricidade (Figura 1). Estes dispositivos são conhecidos como conversores oscilantes por translação de ondas (Oscillating Wave Surge Converter - OWSC) e têm sua hidrodinâmica influenciada pelo grau de submergência de suas placas (WHITTAKER et al., 2007).
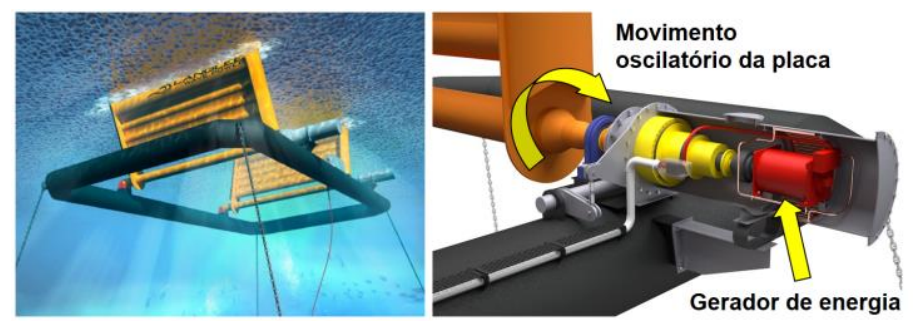

Figura 1 - Princípio de funcionamento de um OWSC

Assim, o presente trabalho está focado no estudo numérico da influência do grau de submergência de um OWSC parcialmente submerso (como o mostrado na Figura 1) sobre seu 


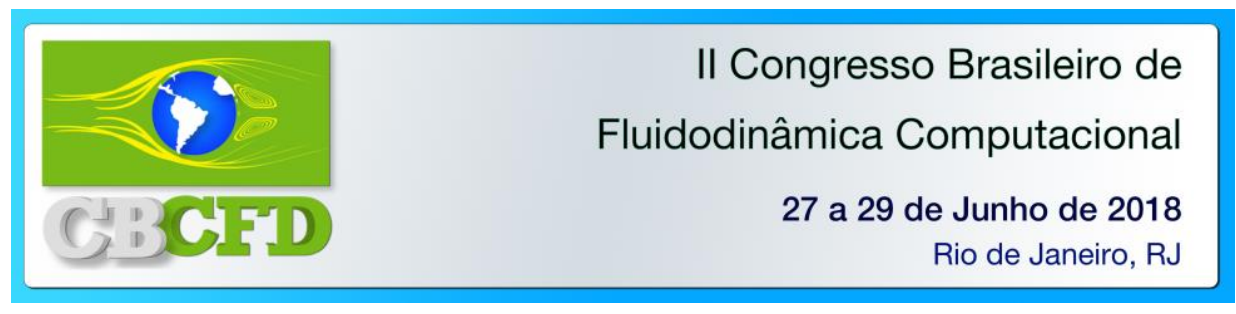

desempenho hidrodinâmico. Para tal, são utilizadas simulações numéricas de um escoamento bifásico, baseadas nas médias de Reynolds (RANS), que utilizam a metodologia VOF Volume of Fluid - (CONDE; CARDOSO, 2016) para o tratamento da superfície livre e a deformação da malha de cálculo para a representação da dinâmica do corpo rígido.

\section{METODOLOGIA}

As simulações numéricas deste trabalho são realizadas pelo código computacional opensource OpenFOAM v. 4.1, que é fundamentado na metodologia de discretização em volumes finitos e que utiliza a extensão OLAFOAM (HIGERA, 2016) para a geração e hidrodinâmica de ondas progressivas.

O escoamento é governado pelas equações da continuidade (Equação 1) e de NavierStokes (Equação 2), descritas em termos médios (CHENG et al., 2003):

$$
\begin{aligned}
& \frac{\partial \bar{u}_{i}}{\partial t}+\bar{u}_{j} \frac{\partial \bar{u}_{i}}{\partial x_{j}}=-\frac{1}{\rho} \frac{\partial \bar{P}}{\partial x_{i}}+\frac{\partial}{\partial x_{j}}\left(v \frac{\partial \bar{u}_{i}}{\partial x_{j}}-\overline{u_{i}^{\prime} u_{j}^{\prime}}\right)+g_{i}, \\
& \frac{\partial \bar{u}_{j}}{\partial x_{j}}=0,
\end{aligned}
$$

em que $u_{i}$ representa a $i$-ésima componente do vetor velocidade, $\mathrm{P}$ a pressão, $x$ a coordenada, $t$ o tempo e $g_{i}$ a aceleração da gravidade na direção i. Para a resolução do tensor de Reynolds utilizou-se a hipótese de Boussinesq e para a modelagem da viscosidade turbulenta foi empregado o modelo de turbulência $\kappa-\omega$ SST (MENTER et al., 2003).

Para o movimento do corpo rígido utilizou-se o solver dinâmico denominado dynamicMotionSolverFvMesh, juntamente com a condição de deformação de malha conhecida como sixDoFRigidBodyDisplacement, responsável por guardar informações da massa e do momento de inércia do objeto considerado, assim como, das restrições e graus de liberdade do movimento do corpo (OPENFOAM WIKI, 2016). A equação que governa o movimento do OWSC é análoga à equação do sistema massa-mola (RENZI et al., 2012):

$$
\mathrm{I}_{\mathrm{m}} \frac{\partial^{2} \theta}{\partial \mathrm{t}}+\mathrm{C} \frac{\partial \theta}{\partial \mathrm{t}}+\mathrm{R} \theta=\mathrm{M}_{\mathrm{t}}
$$

onde $\mathrm{I}_{\mathrm{m}}, \mathrm{C}, \mathrm{R}, \theta \mathrm{e} \mathrm{M}_{\mathrm{t}}$, representam, respectivamente, o momento de inércia de massa do corpo, o coeficiente de amortecimento aplicado ao dispositivo, o momento de restauração causado pelo empuxo, a amplitude angular (medida a partir da posição vertical) e o momento total sobre a placa do conversor.

Utilizou-se uma malha estruturada de elementos com tamanho constante em todas as direções $\left(\Delta_{x}=\Delta_{y}=0,04 \mathrm{~m}\right)$, criada a partir do solver SnappyHexMesh, próprio do código, facilitando o tratamento da superfície livre e da dinâmica do corpo rígido. A discretização temporal é variada a cada passo de tempo (sendo a inicial igual a 0,01 s), em função da 


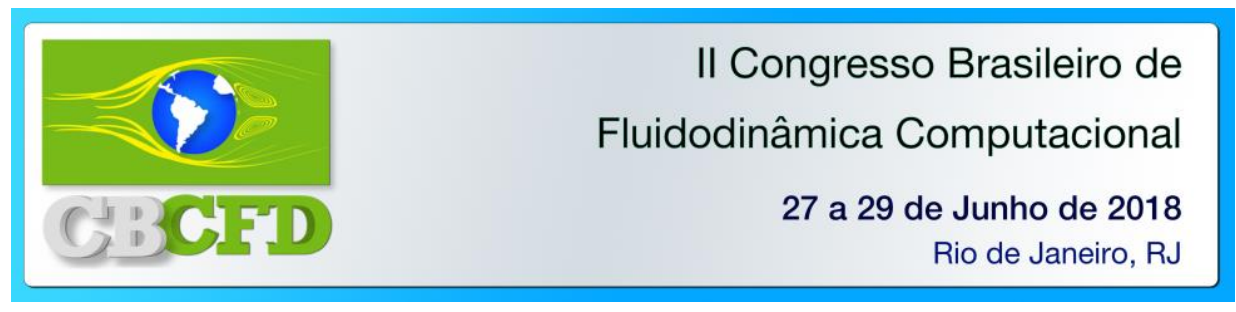

deformação dos elementos de malha, garantindo a convergência e estabilidade das simulações realizadas pelo modelo bidimensional.

O domínio de cálculo e as condições de contorno utilizados estão esquematizados na Figura 2 (onde $h_{\text {sub }}$ e $\mathrm{h}$, representam, respectivamente, a altura de placa submersa e a lâmina d'água), que mostra as configurações e dimensões consideradas. A massa específica e o momento de inércia de massa da placa prismática utilizada foram estimados, respectivamente, em $620,3 \mathrm{~kg} / \mathrm{m}^{3}$ e $0,86 \mathrm{~kg} \cdot \mathrm{m}^{2}$.

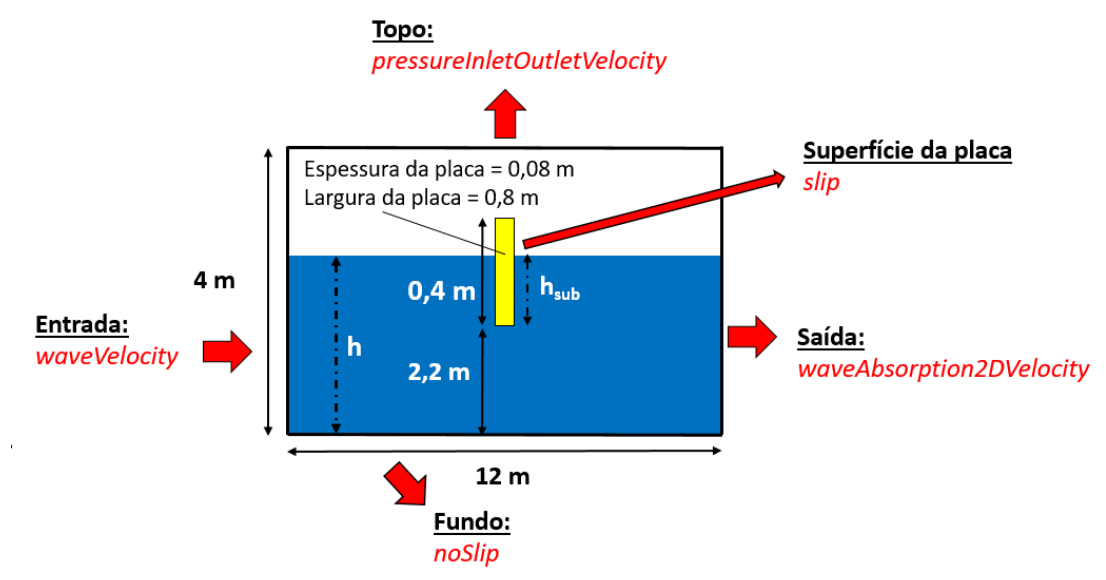

Figura 2 - Domínio de cálculo e condições de contorno utilizados nas simulações

\section{RESULTADOS E CONCLUSÕES}

Para as simulações numéricas realizadas, mantiveram-se fixos a altura $(\mathrm{H})$ e o período de onda (T) em, respectivamente, $0,12 \mathrm{~m}$ e 2,4 s. Assim, variou-se a lâmina d'água (h ) em cinco valores diferentes: $2,48 \mathrm{~m} ; 2,52 \mathrm{~m} ; 2,56 \mathrm{~m} ; 2,60 \mathrm{~m}$ e $2,64 \mathrm{~m}$. Valores estes que representam, em ordem, os seguintes graus $\left(G_{s}\right)$ de submergência (definido como a relação entre a profundidade submersa $\left(\mathrm{h}_{\text {sub }}\right)$ e altura da placa): 0,$7 ; 0,8 ; 0,9 ; 1$ e 1,1 .

A Figura 3a mostra o comportamento do campo de velocidades para os graus de submergência de 1,1, 0,9 e 0,7. Nela é possível perceber grande similaridade entre o padrão de escoamento nas regiões dos entornos das placas oscilantes e, também, que para menores graus de submergência maior será o aumento de velocidades na região próxima à base inferior, enquanto em maiores graus, ocorre uma intensificação da magnitude das velocidades na parte superior da estrutura. Ao comparar estes campos com as curvas resultantes dos valores eficazes (RMS) da amplitude angular e da velocidade angular $\omega$ (Figura 3b) - que são adimensionalizados com base na celeridade (C) da onda incidente - em função do grau de submergência, pode-se concluir que o aumento de velocidades, tanto na parte inferior quanto na superior da placa, é responsável por dificultar a hidrodinâmica dos dispositivos, tendo em vista que as maiores magnitudes ocorridas nos campos de velocidade estão relacionadas aos menores desempenhos hidrodinâmicos dos OWSC's. Por outro lado, o melhor desempenho foi encontrado para casos em que o grau de submergência encontra-se próximo a 0,9 (90\% do dispositivo submerso). 


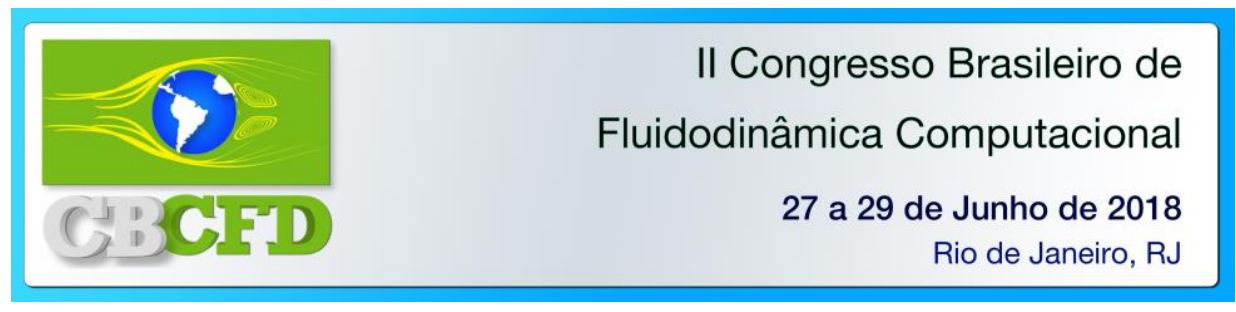

(a)

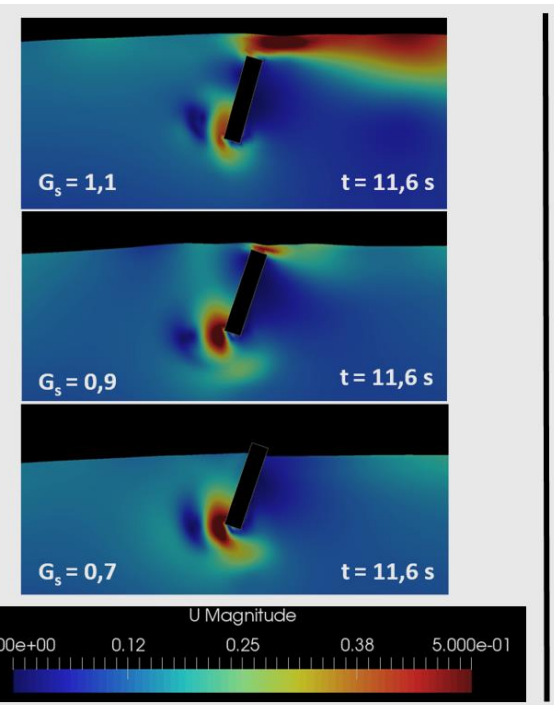

(b)
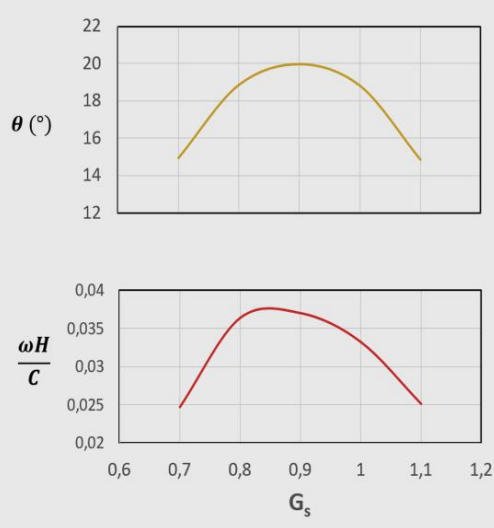

Figura 3 - Campo de velocidades, amplitude angular e velocidade angular em função do grau de submergência do OWSC

\section{REFERÊNCIAS}

Cheng, Y., Lien, F. S., Yee, E., \& Sinclair, R. (2003). A comparison of large eddy simulations with a standard $\mathrm{k}-\varepsilon$ Reynolds-averaged Navier-Stokes model for the prediction of a fully developed turbulent flow over a matrix of cubes. Journal of Wind Engineering and Industrial Aerodynamics, 91(11), 1301-1328.

Conde, J. M. P., \& Cardoso, J. M. S. M. (2016). Numerical Simulation of the Oscillating Water Column inside a Vertical Cylinder in Regular Waves Using IHFOAM. In Defect and Diffusion Forum (Vol. 372, pp. 40-49). Trans Tech Publications.

HIGERA, P. OLAFOAM Reference Manual. Cantabria: Universidad de Cantabria, 2016.

Menter, F. R., Kuntz, M., \& Langtry, R. (2003). Ten years of industrial experience with the SST turbulence model. Turbulence, heat and mass transfer, 4(1), 625-632.

OPENFOAM FOUNDATION. OpenFOAM User Guide v. 4.0. 2016. Disponível em: <http://foam. sourceforge.net/docs/Guides-a4/OpenFOAMUserGuide-A4.pdf >. Acesso em: 21 mar. 2017.

Renzi, E., \& Dias, F. (2012). Resonant behaviour of an oscillating wave energy converter in a channel. Journal of Fluid Mechanics, 701, 482-510.

Whittaker, T., Collier, D., Folley, M., Osterried, M., Henry, A., \& Crowley, M. (2007, September). The development of Oyster - a shallow water surging wave energy converter. In Proceedings of the 7th European Wave and Tidal Energy Conference, Porto, Portugal (pp. 1114). 